\title{
Abordagem fisioterapêutica na Síndrome de Edwards: uma revisão integrativa
}

\section{Physiotherapeutic approach in Edwards Syndrome: an integrative review}

\author{
Juliana Provensi Oro ${ }^{1}$, Matheus Santos Gomes Jorge ${ }^{1}$
}

1 - Universidade de Passo Fundo - UPF, Passo Fundo, RS, Brasil.

\section{RESUMO}

Introdução: a Síndrome de Edwards é uma doença congênita autossômica rara, caracterizada pela trissomia no $18^{\circ}$ par cromossômico. A taxa de mortalidade destes indivíduos é alta e, quando sobrevivem, apresentam complicações físicas e cognitivas graves. A fisioterapia apresenta-se como um dos componentes multidisciplinares essenciais para a qualidade de vida desta população. Objetivo: conhecer as evidências acerca da abordagem fisioterapêutica em indivíduos com Síndrome de Edwards. Método: revisão integrativa da literatura realizada nas bases de dados Embase, Medline, LILACS, Scopus, Web of Science, Cochrane Library, PEDro e SciELO, utilizando os descritores "Trisomy 18 Syndrome" e "Physical Therapy Specialty". Adicionalmente, utilizou-se a palavra-chave "Edwards Syndrome". Dos 35 artigos encontrados, cinco preencheram os critérios de seleção do estudo. Resultados: houve predomínio dos relatos de caso. As abordagens fisioterapêuticas utilizadas foram a hidroterapia, a fisioterapia respiratória,

Palavras-chave: os exercícios de amplitude de movimento, o Método Bobath, exercícios de reeducação/aprendiSíndrome da Trissomia do zagem motora e psicomotricidade e o uso de órteses. Estas intervenções foram benéficas para a Cromossomo 18; Re- regulação dos sinais vitais, alívio da dor, melhora da condição respiratória, dos aspectos motores cém-Nascido Prematuro; Fisioterapia; Hidroterapia; Unidades de Terapia e de aprendizagem, da amplitude de movimento articular, da marcha e da atividade de vida diária para realizar a higiene. Conclusão: os estudos sobre o tema pesquisado são escassos, porém as evidências demonstram a necessidade do tratamento fisioterapêutico para proporcionar qualidade Intensiva Neonatal. de vida aos indivíduos com Síndrome de Edwards.

\section{ABSTRACT}

Introduction: Edwards syndrome is a rare autosomal congenital disease, characterized by trisomy in the 18th chromosomal pair. The mortality rate of individuals with this syndrome is high and, when they survive, they present serious physical and cognitive complications. Physiotherapy is one of the essential multidisciplinary components for better quality of life of this population. Objective: to know the evidence available about the physiotherapeutic approach employed in individuals with Edwards syndrome. Method: an integrative literature review was carried out in the Embase, Medline, LILACS, Scopus, Web of Science, Cochrane Library, PEDro and SciELO databases, using the descriptors "Trisomy 18 Syndrome" and "Physical Therapy Specialty". Additionally, the keywords "Edwards Syndrome" were used. Of the 35 articles found, five met the study selection criteria. Results: there was a predominance of case reports. The physiotherapeutic approaches used were hydrotherapy, respiratory physiotherapy, range of motion exercises,

Keywords: the Bobath concept, motor learning and psychomotricity exercises and the use of orthoses. These Trisomy 18 Syndrome; interventions were beneficial for the regulation of vital signs, pain relief, improvement of the Premature Infant; Phy- respiratory condition, motor and learning aspects, range of articular movement, gait and daily sical Therapy Specialty; life activity to perform hygiene. Conclusion: studies on the researched topic are scarce, but the Hydrotherapy; Neonatal evidence demonstrates the need for physical therapy treatment to provide quality of life to indiIntensive Care Units. viduals with Edwards syndrome. 


\section{INTRODUÇÃO}

A trissomia do cromossomo 18 , foi descrita, em 1960, pelo geneticista John H. Edwards, para caracterizar um indivíduo recém-nascido que apresentava múltiplas malformações congênitas e déficit cognitivo. ${ }^{1}$ Atualmente, também conhecida como Síndrome de Edwards (SE), é identificada como uma doença autossômica, onde há a presença de um cromossomo extra no $18^{\circ}$ par cromossômico. ${ }^{2}$ Pode se manifestar de forma parcial ou em mosaico, ${ }^{3}$ sendo que este último ocorre quando as células com linhagem normal e trissomia no cromossomo 18 se encontram no mesmo indivíduo. ${ }^{4}$

A prevalência da SE é de, aproximadamente, um caso para cada 3 mil a 8 mil nascidos vivos. Todavia, essa taxa aumenta para um caso a cada 2,5 mil a 2,6 mil casos, se considerar os casos de natimortalidade e interrupção voluntária no pré-natal após o diagnóstico. ${ }^{5}$ Os casos de SE do tipo mosaico representam 5\% dos casos ${ }^{4}$. Entre os sexos, a relação é de um indivíduo do sexo masculino acometido para cada dois indivíduos do sexo feminino acometidos. ${ }^{6}$ $\mathrm{Na}$ maioria das trissomias, há uma relação entre a síndrome e a idade materna avançada, ${ }^{7,8}$ sendo este o principal fator para a não disjunção dos cromossomos na fase de divisão celular. ${ }^{9}$

A SE é uma doença genética grave que afeta as funções celulares fetais, causando complicações organogênicas e no desenvolvimento dos tecidos. ${ }^{6}$ $\mathrm{O}$ fenótipo compreende um conjunto de padrões de alterações físicas e deficiência psicomotora e cognitiva, ${ }^{5}$ incluindo cardiopatia congênita (9095\%), comprometimento neurológicos e cognitivos (70\%), artéria umbilical única (80\%), mãos cerradas com sobreposição de $2^{\circ}$ e $3^{\circ}$ dedos $(80 \%)$, pé equino (20\%), onfalocele intestinal (20-25\%) e alterações no perímetro cefálico $(43 \%) .{ }^{10,11}$ Desta forma, tais complicações contribuem significativamente para a elevada taxa de mortalidade e morbidade infantil e neonatal destes indivíduos. ${ }^{5}$

Em razão das complicações clínicas causadas pela SE, a fisioterapia busca manter sua capacidade funcional e proporcionar bem-estar e qualidade de vida para estes indivíduos. ${ }^{12}$ Dentre as técnicas utilizadas, pode-se citar a pressão positiva contínua nas vias aéreas (CPAP) e o Método Bobath. ${ }^{13}$ No entanto, na literatura, os estudos são consistentes quanto ao tratamento fisioterapêutico na Síndrome de Down ${ }^{14,15}$ e carece de publicações em outras síndromes congênitas, como é o caso da SE. Desta forma, conhecer o quadro clínico e o prognóstico dos indivíduos com esta patologia é de extrema valia no norteamento dos cuidados neonatais e na tomada de decisão do tratamento, seja ele invasivo ou não. ${ }^{16} \mathrm{O}$ objetivo do presente estudo foi conhecer as evidências acerca da abordagem fisioterapêutica em indivíduos com SE.

\section{MÉTODO}

Trata-se de um estudo de revisão integrativa da literatura, cuja mesma realizamos de acordo com a orientação para revisões integrativas confiáveis publicadas no estudo de Souza, Silva e Carvalho (2010). ${ }^{17}$

Os critérios de inclusão foram para estudos que envolviam exclusivamente indivíduos com diagnóstico de $\mathrm{SE}$ e estudos que realizaram intervenções fisioterapêuticas na SE, tais como cinesioterapia, abordagem do desenvolvimento motor, fisioterapia respiratória, hidroterapia, entre outras. Os critérios de exclusão foram para estudos observacionais (coorte, caso-controle e transversal), estudos realizados com indivíduos diagnosticados com outras síndromes congênitas, tais como Síndrome de Down, Síndrome de Patau e Síndrome de Turner, e estudos que envolveram abordagem médica, medicamentosa, nutricional, odontológica, cirúrgica ou de qualquer outra área, exceto a fisioterapia.

A estratégia de pesquisa foi realizada por dois revisores, em pares, em 20 de setembro de 2020 , utilizando termos da língua inglesa que estivessem de acordo com o MeSH (Medical Subject Headings). Os descritores utilizados foram "Trisomy 18 Syndrome" e "Physical Therapy Specialty". Adicionalmente, utilizou-se a palavra-chave "Edwards' Syndrome". A busca não restringiu os estudos por língua, período de publicação ou tipo de acesso (livre ou restrito). As bases de dados consultadas foram Embase, Medline (via PubMed), Literatura Latino-americana e do Caribe em Ciências da Saúde (LILACS - via BIREME), Scopus, Web of Science, Cochrane Library, Physioterapy Evidence Database (PEDro) e Scientific Electronic Library Online (SciELO).

Inicialmente, identificaram-se os estudos nas bases de dados e excluiu-se aqueles duplicados (Fase de Identificação). Na sequência, dois revisores analisaram os títulos, resumos e os descritores/ palavras-chaves, sendo que em caso de divergência 
seria requisitado um terceiro avaliador para desempate (Fase de Triagem). Após, os textos completos foram revisados e analisados na íntegra, seguindo os mesmos princípios da fase anterior (Fase de Elegibilidade). Por fim, extraíram-se os dados dos artigos incluídos, tais como suas referências, características amostrais, protocolos metodológicos e desfechos/resultados (Fase de Inclusão) (Figura 1).

\section{RESULTADOS}

De acordo com a nacionalidade dos estudos analisados, observamos que dois estudos eram provenientes do Brasil, ${ }^{18,19}$ um da Austrália, ${ }^{20}$ um do Peru $^{21}$ e um dos Estados Unidos. ${ }^{22}$ Dois dos estudos foram redigidos em português, ${ }^{18,19}$ dois em inglês ${ }^{20,22}$ e um em espanhol. ${ }^{21}$ Houve predomínio de relatos de caso, ${ }^{18-22}$ sendo que houve predomínio do sexo feminino. ${ }^{19-22}$ Dentre os estudos analisados observouse que como condutas fisioterapêuticas foram utilizados a hidroterapia, ${ }^{19}$ a fisioterapia respiratória, ${ }^{18}$ os exercícios de amplitude de movimento ${ }^{20}$, a Método Bobath $^{20}$, exercícios de reeducação/aprendizagem motora e psicomotricidade ${ }^{21,22}$ e o uso de órteses..$^{22}$ Os desfechos apontaram que a intervenção fisioterapêutica foi benéfica para regulação dos sinais vitais e diminuição do quadro doloroso, ${ }^{19}$ a melhora da condição respiratória, ${ }^{18}$ dos aspectos motores e de aprendizagem, ${ }^{20,22}$ da amplitude de movimento articular, ${ }^{20}$ da marcha e da atividade de vida diária para realizar a higiene ${ }^{21}$ (Tabela 1).

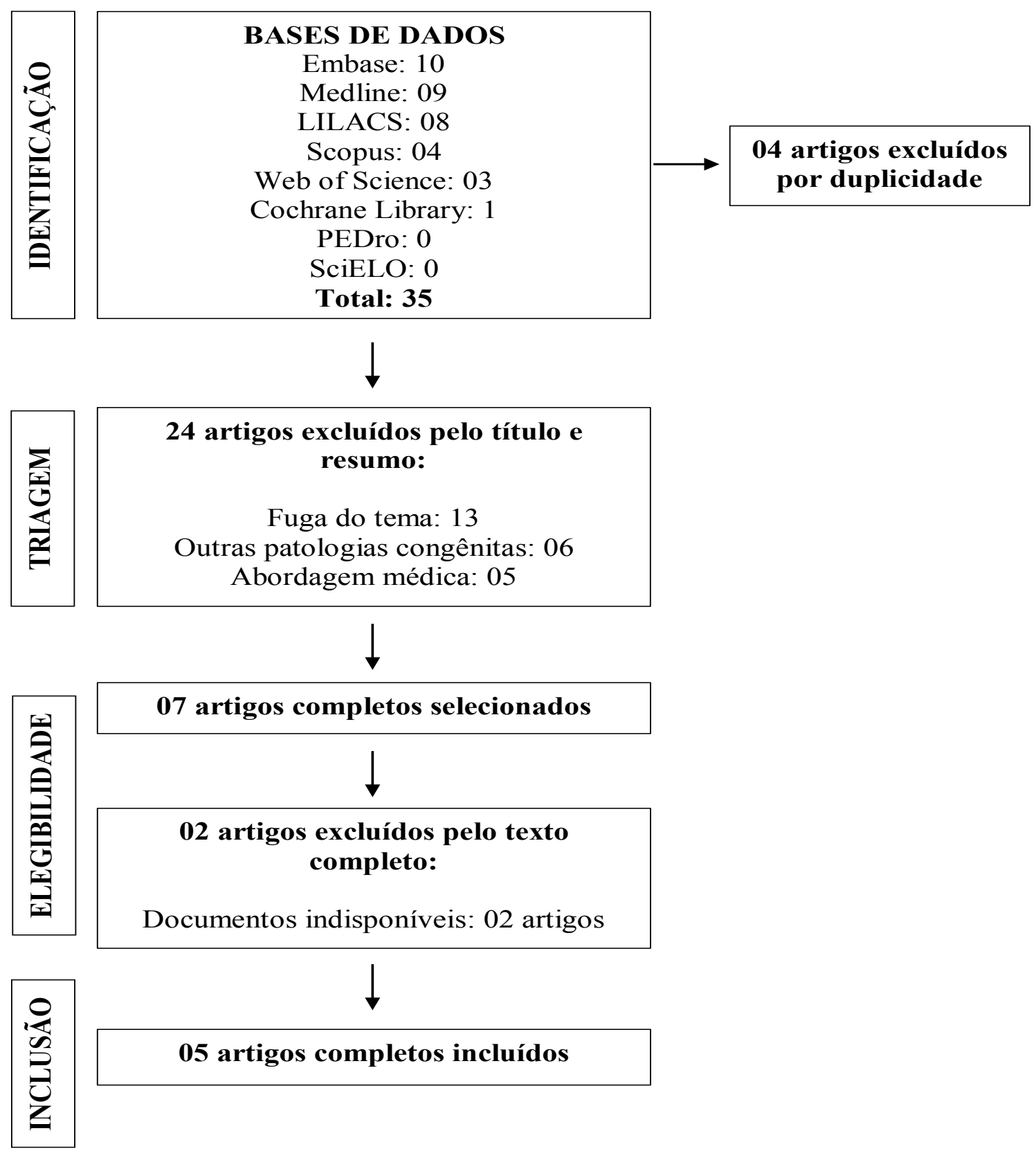




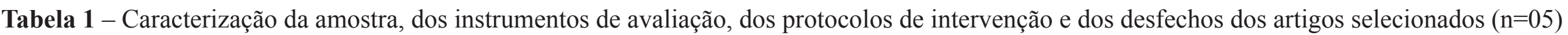

\begin{tabular}{|c|}
\hline Tipo d \\
\hline
\end{tabular}

Abbie, $1976^{20}$ Estudo de Feminino 04 anos

caso

Araújo, $2015^{18}$ Relato de Masculino Recémcaso nascido

Bustillos - Estudo de Feminino Villalta e caso

Quiñones-

$\mathrm{C}$ a $\mathrm{m} \mathrm{p}$ o $\mathrm{s}$,

$2014^{21}$

Guimarães Estudo de Feminino e Barbosa, caso $2009^{19}$ J o h n s o n, caso $1994^{22}$

15 anos
Relatar o desenvolvimento de uma criança com Síndrome de Edwards. de

amplitude

de Dois anos e quatro meses: sem equilibro sentada, mas conseguia Método Bobath para a facilitação de habilidades de desenvolvimento.

Analisar os benefícios da O paciente foi submetido a fisioterapia fisioterapia respiratória e respiratória com mudança no modo suas repercussões clínicas ventilatório

em uma criança com

Síndrome de Edwards

internada na Unidade

de Terapia Intensiva

Neonatal e com intubação

orotraqueal.

Descrever o caso de uma Exercícios de reeducação motora A paciente passou a realizar a marcha independente com uso de adolescente de portadora e exercícios de psicomotricidade, sapato ortopédico e realiza atividades de higiene menores.

da Síndrome de Edwards. controle respiratório e esquema corporal.

02 meses Descrever os benefícios A paciente realizou sete sessões Os sinais vitais melhoraram após a intervenção fisioterapêutica, da hidroterapia em uma de hidroterapia, durante o período onde observou-se aumento da saturação de oxigênio, diminuição criança com Síndrome de de internação hospitalar, incluindo da frequência cardíaca e respiratória (valores dentro da Edwards.

alongamentos, dissociações de tronco e mobilizações articulares.

Descrever o caso de Aprendizagem motora

e Adquiriu habilidades de sequência de movimentos, controle da uma criança com psicomotricidade e uso de órteses cabeça na posição prona, capacidade de andar com o andador, com Síndrome de Edwards toracolombar e calcanhar. assistência para evitar obstáculos e mudar de direção. Permanece submetida às intervenções fisioterapêuticas. normalidade para a idade) e diminuição da dor da paciente (a paciente encontrava-se mais calma e relaxada).

(eis meses: conseguia rolar de decubito dorsal para decúbito se agarrando-se às barras da cama; Dois anos e oito meses: conseguia rolar de decúbito dorsal para decúbito ventral; Quatro diminuição do risco de complicações pulmonares do paciente, uma vez que houve diminuição dos sinais de desconforto respiratório e otimização da ausculta pulmonar. em pé, apoiando-se nos móveis e não mantém as mãos cerradas e dedos sobrepostos. 


\section{DISCUSSÃO}

Oobjetivo desta revisão integrativa da literatura foi conhecer as evidências acerca da abordagem fisioterapêutica em indivíduos com SE. Observamos que as técnicas fisioterapêuticas (hidroterapia, fisioterapia respiratória, exercícios de amplitude de movimento articular, Método Bobath, exercícios de reeducação/aprendizagem motora e psicomotricidade e o uso de órteses) foram benéficas para a regulação dos sinais vitais, alívio da dor, melhora da condição respiratória, dos aspectos motores e de aprendizagem, da amplitude de movimento articular, da marcha e das atividades de vida diária para realizar a higiene das crianças com SE.

A SE é uma doença cromossômica, com alta mortalidade fetal e neonatal. A maioria dos nascidos vivos apresenta baixo índice de Apgar e baixo peso. As deformidades cardíacas congênitas são características da patologia e identificadas, ainda, no estágio prénatal $^{23}$. As características fenotípicas comumente presentes na SE são múltiplas, podendo compreender comprometimentos neurológicos (neuromotores e cognitivos), deficiência mental, hipotonia seguida de hipertonia, alteração do crescimento, alterações do perímetro e morfologia do crânio, da face, do tórax, do abdome e das extremidades dos membros (caracterizada pelas mãos cerradas e sobreposição do segundo e terceiro dedos), além de alterações cutâneas e fâneros, e malformações dos órgãos internos e da região genital. ${ }^{16}$ Neste sentido, a fisioterapia tornase essencial na assistência aos sujeitos com esta síndrome.

A criança com SE deve ser integrada à sociedade, tendo como direito assistência ao cuidado integral e a promoção de saúde. Assim, a fisioterapia para estes indivíduos propõe manter sua capacidade funcional e proporcionar bem-estar e qualidade de vida, ${ }^{12}$ utilizando técnicas fisioterapêuticas como a pressão positiva contínua nas vias aéreas (CPAP) e o Método Bobath. ${ }^{13}$ Em vista disso, estes indivíduos são beneficiados com a fisioterapia. Todavia, poucos estudos envolvem essa temática, possivelmente, pela alta taxa de natimortalidade que ocorre nesta população. ${ }^{23}$

O Método Bobath utiliza exercícios que estimulam a transferência de peso para o indivíduo obter maior controle proprioceptivo e noção de espaço ${ }^{24}$, além de evitar sinergias musculares anormais, reduzir o tônus anormal e facilitar a realização de atividades funcionais ${ }^{25}$. Este método pode ser realizado apenas com toque tato manual do terapeuta ou com auxílio de materiais diversos como bolas suíças, rolos terapêuticos, etc.. ${ }^{24}$

Durante a abordagem do Método Bobath ocorre a ativação do controle motor por meio do "mecanismo reflexo postural normal", cujo mesmo é produto da interação entre as reações de equilíbrio e retificação. Com isso, o indivíduo adquire o controle motor e, consequentemente, a capacidade de realizar o movimento. As diretrizes do Método Bobath são delineadas para diversos tipos de quadros patológicos, especialmente de indivíduos com alguma disfunção neurológica, ${ }^{26}$ como é o da $\mathrm{SE}$, onde o comprometimento neurológico ocorre em quase $100 \%$ dos casos. Isso corrobora com a literatura, cuja mesma aponta que a maioria dos fisioterapeutas utiliza este método como uma das principais intervenções no tratamento de crianças com doenças congênitas, com tempo de intervenção em torno de 30 minutos e com frequência de uma a duas vezes semanais. ${ }^{27}$

Neste sentido, um dos estudos recrutados ${ }^{20}$ utilizou o Método Bobath em uma criança com SE, uma doença congênita e rara, que causa comprometimentos neurológicos, e observou melhora do desenvolvimento motor do indivíduo. Isto vai ao encontro de uma pesquisa, onde observou-se que terapias combinadas, incluindo o Método Bobath, por um tempo intensivo, foi capaz de melhorar a capacidade de realizar tarefas de crianças com atraso no desenvolvimento motor. ${ }^{28}$

O processo de engatinhar se dá quando o bebê, ao tentar mover o tronco para frente, utiliza a força dos membros superiores para erguer-se e manter o abdômen suspenso no ar, distribuindo o peso corporal nas mãos e nos joelhos. Por fim, o mesmo realiza movimentos coordenados e alternados entre os membros superiores e inferiores que permitam-no rastejar e realizar a ação desejada. ${ }^{29}$

Em uma série de casos realizada com quatro indivíduos com Síndrome de Down submetidos ao Método Bobath observou-se que os mesmos apresentaram evolução das atividades, especialmente na postura prono que exerce grande influência durante a atividade de engatinhar. ${ }^{30}$ Acredita-se que isso se deve em virtude de que um dos objetivos do Método Bobath é aumentar o número de unidades motoras recrutadas e, consequentemente, aumentar a força muscular ${ }^{31}$, melhorando o equilíbrio e o 
controle postural durante este processo. ${ }^{32}$ Desta forma, podemos compreender que o Método Bobath é benéfico para melhorar o desempenho do processo de desenvolvimento motor de crianças com patologias congênitas, como é o caso da SE.

Um estudo de caso realizado com um paciente, com um ano de idade, diagnosticado com Síndrome de Cornelia de Lange, uma doença congênita rara, assim como a SE, evidenciou que o plano fisioterapêutico baseado no Método Bobath, técnicas de inibição reflexa, Método de Colles, além da abordagem multidisciplinar, produziu pequenos avanços motores na criança, tais como, o apoio de cabeça, a abertura das mãos e o rastreamento de objetos com o olhar, ${ }^{33}$ indo ao encontro de um dos estudos recrutados, ${ }^{20}$ que utilizou abordagens associados ao Método Bobath.

Os acometimentos respiratórios são uma das principais complicações na SE, uma doença ligada a prematuridade dos seus acometidos. ${ }^{16} \mathrm{Um}$ estudo retrospectivo, onde analisou-se os prontuários de 172 recém-nascidos prematuros, observou-se que apenas $81 \%$ das mães realizou o pré-natal. A maioria dos bebês nascidos era do sexo feminino (58\%), sendo que $44 \%$ apresentaram acometimentos respiratórios e $24 \%$ neurológicos. Além disso, 63\% dos pacientes internados realizaram fisioterapia respiratórias e motoras. ${ }^{34}$ Desta forma, justifica-se o porquê de indivíduos com SE necessitarem realizar fisioterapia respiratória e motora na UTI neonatal, pois apresenta forte vínculo com a prematuridade e comprometimentos neurológicos e respiratórios.

A fisioterapia neonatal trabalha com a execução de condutas fisioterapêuticas voltadas ao sistema musculoesquelético e cardiorrespiratório. Dentre as técnicas, pode-se citar à manutenção das vias aérea, o gerenciamento da ventilação mecânica, a oxigenioterapia, o suporte ventilatório não invasivo e a mobilização precoce com aplicação de técnicas cinético-funcionais ${ }^{35,36}$. Desta forma, um dos estudos incluídos ${ }^{18}$ realizou a fisioterapia em um neonato com a SE na Unidade de Terapia Intensiva, observando que houve melhora do quadro respiratório, diminuição do risco de complicações pulmonares e dos sinais de desconforto respiratório e otimização da ausculta pulmonar. Contrapartida, a fisioterapia não foi capaz de melhorar os aspectos motores em virtude do próprio quadro evolutivo da doença.

Durante a prática clínica nas UTIs neonatais, rotineiramente são avaliados os indicadores comportamentais da dor para verificar a frequência de sua ocorrência, mesmo após os procedimentos de fisioterapia respiratória. ${ }^{37} \mathrm{~A}$ dor é uma experiência sensorial desagradável que pode ser combatida com o uso simultâneo de abordagens farmacológicas e não farmacológicas, estas últimas incluem procedimentos comumente realizados na rotina fisioterapêutica, como intervenções táteis, massagens e banhos de imersão, promovendo alívio da dor e diminuição do estresse e da agitação do neonato ${ }^{38}$. Isso vem ao encontro de um dos estudos incluídos nesta revisão, ${ }^{19}$ cujo mesmo abordou a fisioterapia aquática em um indivíduo com SE e apresentou alívio dos sinais e sintomas dolorosos, sendo, assim, as condutas fisioterapêuticas uma boa opção para a qualidade de vida do recémnascido com SE.

Reforçando os achados anteriores sobre os benefícios da hidroterapia sobre a dor em recémnascidos, uma pesquisa realizada com 12 recémnascidos clinicamente estáveis, com idade gestacional inferior a 36 semanas, internados em unidade de terapia intensiva neonatal, foram submetidos a intervenção fisioterapêutica por meio da hidrocinesioterapia, com duração de 10 minutos, na qual foram realizados movimentos que estimulam as posturas flexoras e a organização postural. Após a fisioterapia, os recémnascidos apresentaram melhora do estado de sonovigília $(p<0,001)$ e diminuição dos escores da escala de avaliação de dor $(p<0,001)$, com manutenção dos valores dos sinais vitais, sugerindo que a fisioterapia aquática pode ser um método simples e efetivo na redução da dor e na melhora da qualidade do sono destes indivíduos ${ }^{38}$. Neste sentido, podemos estender estes benefícios para os indivíduos prematuros acometidos pela SE.

Uma das técnicas da fisioterapia aquática é o banho de imersão, capaz de diminuir a sensibilidade das terminações nervosas, aumentar a circulação sanguínea da musculatura periférica e respiratória com consequente, melhora da capacidade vital. Isto promove uma sensação de bem-estar ao bebê, relaxando os músculos e aliviando o quadro doloroso. ${ }^{39} \mathrm{Um}$ estudo realizado com a finalidade de avaliar os efeitos da hidroterapia sobre os sinais vitais (frequência cardíaca, frequência respiratória, saturação de oxigênio e dor, este último mensurado pela Escala NIPS) de dez recém-nascidos prematuros hospitalizados, demonstrou que, após a intervenção, houve um aumento da saturação de oxigênio e redução 
dos valores da frequência cardíaca, da frequência respiratória e do nível de dor ${ }^{40}$. Indo ao encontro de um dos estudos incluídos ${ }^{19}$ em nossa revisão, onde aplicação da hidroterapia em um recém-nascido com SE, obteve resultados semelhantes.

Ensaio clínico não randomizado avaliou a eficácia da hidroterapia sobre o stress em 15 prematuros. Durante 15 dias, foram avaliados os sinais vitais, o nível de dor e os níveis de cortisol pela saliva dos indivíduos, antes e após cada sessão de 10 minutos. Após os 15 dias, observou-se redução dos níveis de cortisol, diminuição da frequência respiratória e cardíaca, e aumento da saturação de oxigênio, porém com pouca mudança no quadro álgico dos indivíduos. ${ }^{41}$ Isso vai ao encontro ao nosso estudo, onde observamos que a hidroterapia foi uma técnica benéfica para diminuição dos parâmetros hemodinâmicos e alívio da dor em indivíduos com SE. ${ }^{19}$ Em suma, a fisioterapia aquática é tida como um método favorável e seguro para bebês e crianças, proporcionando melhora do desenvolvimento neuromotor, bem-estar e alívio da dor. A aplicação da técnica deve ser feita com profissional capacitado que esteja atento a aspectos fundamentais para o sucesso da terapêutica como os sinais vitais do paciente, a temperatura da água e a assepsia do local. ${ }^{39}$

O nosso estudo apresentou algumas limitações. Durante a busca, identificamos artigos que elegíveis para integrar o componente teórico da revisão, porém, os textos na íntegra dos mesmos não foram localizados e estes foram excluídos. Apesar disso, isso não invalida a geração de dados e formação do conhecimento acerca dos efeitos da fisioterapia para indivíduos com SE.

A sintetização de nosso estudo sobre o tratamento fisioterapêutico na SE, pode auxiliar no delineamento da reabilitação com abordagens mais assertivas, visto que a exploração deste tema apresenta uma expressiva carência científica. Embora não tenhamos investigado as causas para tal escassez, acreditamos que isto possa estar relacionado a raridade da doença. Além disso, este estudo pode contribuir na elaboração de intervenções qualificadas para melhorar a qualidade de vida desta população, bem como esclarecer possíveis dúvidas que os fisioterapeutas enfrentem na assistência ao paciente com SE, cujo mesmo apresenta um quadro complexo e, muitas vezes, delicado.

\section{CONCLUSÃO}

Em virtude da raridade da doença e da baixa expectativa de vida, os estudos que envolvem o tratamento fisioterapêutico na SE são escassos e necessitam de maior aprofundamento. Apesar disso, os métodos utilizados em fisioterapia, tais como hidroterapia, fisioterapia respiratória, exercícios de amplitude de movimento, Método Bobath, exercícios de reeducação/aprendizagem motora e psicomotricidade e o uso de órteses, são benéficos para regulação dos sinais vitais, diminuição da dor, melhora da condição respiratória, dos aspectos motores e de aprendizagem, da amplitude de movimento articular, da marcha e das atividades de vida diária em indivíduos com SE.

\section{REFERÊNCIAS}

1. Edwards JH, Harnden DG, Cameron AH, Crosse VM, Wolf OH. A new trisomic syndrome. Lancet 1960;275(7128):787-90. https://doi. org/10.1016/S0140-6736(60)90675-9

2. Saldarriaga W, Rengifo-Miranda H, Ramírez-Cheyne J. Síndrome de trisomía 18. Reporte de un caso clínico. Rev Chil Pediatría 2016;87(2):129-36. doi: https://doi.org/10.1016/j.rchipe.2015.08.006

3. Tucker ME, Garringer HJ, Weaver DD. Phenotypic spectrum of mosaic trisomy 18: Two new patients, a literature review, and counseling issues. Am J Med Genet Part A 2007;143(5):505-17. doi: https://doi. org/10.1002/ajmg.a.31535

4. Motta S, Sala D, Sala A, Cazzaniga G, Giudici G, Villa N, et al. Hodgkin lymphoma in a patient with mosaic trisomy 18: First clinical observation. Am J Med Genet Part A 2016;170(3):777-80. doi: https:// doi.org/10.1002/ajmg.a.37504

5. Mudaliyar US, Mudaliyar SU. Strawberry skull in Edwards syndrome. BJR|Case Reports 2017;3(4):1-3. doi: https://doi.org/10.1259/ bjrcr.20170045

6. Crawford D, Dearmun A. Edwards' Syndrome. Nurs Child Young People 2016;28(10):1-17. doi: https://doi.org/10.7748/ncyp.28.10.17. s19

7. Lin H-Y, Lin S-P, Chen Y-J, Hung H-Y, Kao H-A, Hsu C-H, et al. Clinical characteristics and survival of trisomy 18 in a medical center in Taipei, 1988-2004. Am J Med Genet Part A 2006;140(9):945-51. doi: https://doi.org/10.1002/ajmg.a.31173

8. Rosa RFM, Rosa RCM, Lorenzen MB, Moraes FN, Graziadio C, Zen PRG, et al. Trisomy 18: Experience of a reference hospital from the south of Brazil. Am J Med Genet Part A 2011;155(7):1529-35. doi: https://doi.org/10.1002/ajmg.a.34088

9. Bugge M, Collins A, Petersen MB, Fisher J, Brandt C, Hertz JM, et al. Non-disjunction of chromosome 18. Hum Mol Genet 1998;7(4):661-9. doi: https://doi.org/10.1093/hmg/7.4.661

10. Bromley B. Diagnostic Imaging of Fetal Anomalies. Nyberg DA, McGahan JP, Pretorius DH, Pilu G, editors. Vol. 22, Journal of Ultrasound in Medicine. Philadelphia: Lippincott Williams \& Wilkins; 2003. 850-850 p. doi: https://doi.org/10.7863/jum.2003.22.8.850

11. Yonehara T, Hata T, Aoki S. Three-dimensional sonography in diagnosing trisomy 18. Am J Roentgenol 1998;171(4):1165-6. doi: https://doi.org/10.2214/ajr.171.4.9763024

12. Goretti T, Carvalho ML de, Pimentel GM. Integralidade do cuidado e a promoção da saúde de uma criança com Síndrome de Edwards: um estudo de caso. Cad Educ Saúde e Fisioter 2014;1(1):1-1. 
13. Santos CCT, Barcellos C de P, Tolentino L, Silva LA da, Romariz TP. Criança com síndrome de Edwards, abordagem fisioterapêutica. Reabilitar 2004;6(1):46-9.

14. Eid MA, Aly SM, Huneif MA, Ismail DK. Effect of isokinetic training on muscle strength and postural balance in children with Down's syndrome. Int J Rehabil Res 2017;40(2):127-33. doi: https:// doi.org/10.1097/MRR.0000000000000218

15. Miguel A de, Miguel MD de, Lucena-Antón D, Rubio MD. Efectos de la hipoterapia sobre la función motora en personas con síndrome de Down: revisión sistemática. Rev Neurol 2018;67(7):233-41. doi: https://doi.org/10.33588/rn.6707.2018117

16. Rosa RFM, Rosa RCM, Zen PRG, Graziadio C, Paskulin GA. Trissomia 18: revisão dos aspectos clínicos, etiológicos, prognósticos e éticos. Rev Paul Pediatr 2013;31(1):111-20. doi: https://doi. org/10.1590/S0103-05822013000100018

17. Souza MT de, Silva MD da, Carvalho R de. Integrative review: what is it? How to do it? Einstein (São Paulo) 2010;8(1):102-6. doi: https://doi.org/10.1590/s1679-45082010rw1134

18. Araújo ACF. Atuação da fisioterapia respiratória na Síndrome de Edwards em uma Unidade de Terapia Intensiva Neonatal em Santos SP - relato de caso. Rev UNILUS Ensino e Pesqui 2015;12(1):42-4.

19. Guimarães DB, Barbosa LPC. Os benefícios da hidroterapia na síndrome de Edwards: relato de caso. Pediatria (Santiago) 2009;31(2):137-40.

20. Abbie M. Unusual Development of Motor Skills in a Child with Trisomy-18. Dev Med Child Neurol 1976;18(1):85-9. doi: https://doi. org/10.1111/j.1469-8749.1976.tb03608.x

21. Bustillos-Villalta K, Quiñones-Campos M. Síndrome de Edwards de larga supervivencia: efecto del tratamiento rehabilitador integral. Rev Med Hered 2014;25(1):89-92.

22. Woldorf JW, Johnson K. Gross Motor Development of a 7-YearOld Girl With Trisomy 18. Clin Pediatr (Phila) 1994;33(2):120-2. doi: https://doi.org/10.1177/000992289403300211

23. Denardin D, Savaris FE, Cunha AC da, Betat R da S, Telles JAB, Targa LV, et al. Retrospective cohort of trisomy 18 (Edwards syndrome) in southern Brazil. Sao Paulo Med J 2014;133(4):320-5. doi: https:// doi.org/10.1590/1516-3180.2013.79900715

24. Silva TF. A Importância do Método Bobath na reabilitação de criança com paralisia cerebral. Rev Científica Multidiscip Núcleo do Conhecimento. 2017;11(1):15-23.

25. Oliveira L dos S de, Golin MO. Técnica para redução do tônus e alongamento muscular passivo: efeitos na amplitude de movimento de crianças com paralisia cerebral espástica. ABCS Heal Sci 2017;42(1):27-33.

26. Bobath B. Desenvolvimento motor dos diferentes tipos de paralisia cerebral. São Paulo: Manoele; 1978. 124 p.

27. Morais KDW de, Fiamenghi-Jr GA, Campos D, Blascovi-Assis SM. Profile of physiotherapy intervention for Down syndrome children. Fisioter em Mov 2016;29(4):693-701. doi: https://doi. org/10.1590/1980-5918.029.004.ao05

28. Russell DC, Scholtz C, Greyling P, Taljaard M, Viljoen E, Very C. A pilot study on high dosage intervention of children with $\mathrm{CP}$ using combined therapy approaches. South African J Occup Ther 2018;48(2):26-33. doi: https://doi.org/10.17159/2310-3833/2017/ vol48n2a5

29. Xiong QL, Hou WS, Xiao N, Chen YX, Yao J, Zheng XL, et al. Motor Skill Development Alters Kinematics and Co-Activation Between Flexors and Extensors of Limbs in Human Infant Crawling. IEEE Trans Neural Syst Rehabil Eng 2018;26(4):780-7. doi: https://
doi.org/10.1109/TNSRE.2017.2785821

30. Santos GR dos, Cabral LC, Silva LR, Dionisio J. Physiotherapeutic stimulation in infants with Down syndrome to promote crawling. Fisioter em Mov 2020;33(1):1-9. doi: https://doi.org/10.1590/19805918.033.ao54

31. Firmino R, Lima AK, Almeida C, Uchôa S. Influence of Bobath's Concept on muscle function of the spastic tetraplegia cerebral palsy. Rev Neurociências 2015;23(4):595-602. doi: https://doi.org/10.4181/ RNC.2015.23.04.1047.08p

32. Tekin F, Kavlak E, Cavlak U, Altug F. Effectiveness of NeuroDevelopmental Treatment (Bobath Concept) on postural control and balance in Cerebral Palsied children. J Back Musculoskelet Rehabil 2018;31(2):397-403. doi: https://doi.org/10.3233/BMR-170813

33. Rodríguez, Lis Lorena Luis YRC, Labrada, Rolando Michel Llópiz DLL. Tratamiento rehabilitador a un paciente con síndrome de Cornelia de Lange. Correo Científico Médico de Holguín 2017;21(1):932-8.

34. Pedro FKS, Torati CV, Gentilli R de ML, Sogame LCM. Assistência fisioterapêutica em recém-nascidos prematuros internados em UTI neonatal pública. Fisioter Bras 2013;14(1):99-105.

35. Zhou B, Zhai J-F, Wu J-B, Jin B, Zhang Y-Y. Different ventilation modes combined with ambroxol in the treatment of respiratory distress syndrome in premature infants. Exp Ther Med 2017;13(2):629-33. doi: https://doi.org/10.3892/etm.2016.3978

36. Kessler RMG, Alcará LP, Barduzzi Netto TVL. Revisão integrativa: fisioterapia em terapia intensiva neonatal. Rev Interdiscip Estud em Saúde 2019 Nov;8(2):227-38. doi: https://doi.org/10.33362/ries. v8i2.1560

37. Martins R, Silva MEM da, Honorio GJ da S, Paulin E, Schivinski CIS. Tecnicas de fisioterapia respiratoria: efeito nos parametros cardiorrespiratorios e na dor do neonato estável em UTIN. Rev Bras Saúde Matern Infant 2013;13(4):317-27. doi: https://doi.org/10.1590/ S1519-38292013000400004

38. Vignochi C, Teixeira PP, Nader SS. Efeitos da fisioterapia aquática na dor e no estado de sono e vigília de recém-nascidos pré-termo estáveis internados em unidade de terapia intensiva neonatal. Rev Bras Fisioter 2010;14(3):214-20. doi: https://doi.org/10.1590/S141335552010000300013

39. Rambo DC, De Souza AQ, Kruel CS, Filippin NT. Fisioterapia aquática aplicada em recém-nascidos e crianças: uma revisão integrativa. Rev Eletrônica Acervo Saúde 2019;1(30):1-8. doi: https:// doi.org/10.25248/reas.e728.2019

40. Barbosa LPC, Carneiro ÉM, Weffort V. Impacto da hidroterapia em recém-nascidos hospitalizados. Fisioter Bras 2015;16(1):207-11.

41. Tobinaga WC de O, Marinho C de L, Abelenda VLB, de Sá PM, Lopes AJ. Short-Term Effects of Hydrokinesiotherapy in Hospitalized Preterm Newborns. Rehabil Res Pract 2016;2016(1):1-8. doi: https:// doi.org/10.1155/2016/9285056

Como citar: ORO, Juliana Provensi; JORGE, Matheus Santos Gomes. Abordagem fisioterapêutica na Síndrome de Edwards: uma revisão integrativa. Revista Interdisciplinar de Promoção da Saúde, Santa Cruz do Sul, v. 3, n. 3, jul 2020. ISSN 25953664. Disponível em: <https://online.unisc.br/seer/index.php/ripsunisc/article/view/16203>. Acesso em: 01 jul 2020 . doi:https:// doi.org/10.17058/rips.v3i3.16203 